\title{
Ende des Abwärtstrends? Zur Entwicklung des Streikvolumens in Westeuropa seit Beginn der Weltwirtschaftskrise
}

Die wirtschaftliche Entwicklung seit 2008 wird von einer neuen sozialen Protestwelle begleitet, über die damit verbundene Streikaktivität ist bislang jedoch wenig bekannt. Das wirft die Frage auf, was die Streikstatistik über die Folgen der Wirtschaftskrise aussagen kann. Aus Presse- und Medienberichten geht klar hervor, dass politische Massenstreiks im Zuge von Wirtschaftskrise und Austeritätspolitik zunehmen. Aber sind politische Massenstreiks die einzige Erklärung für die auffallenden Spitzen in der Streikaktivität? Bedeuten politische Massenstreiks ferner wirklich, dass in keinem westeuropäischen Land mehr friedliche Arbeitsbeziehungen vorherrschen? Und schließlich: Bleiben Streiks auch weiterhin zentrale Instrumente im Arsenal für den Arbeitskampf? ${ }^{1}$

KURT VANDAELE

\section{Einführung}

Gesellschaftliche Mobilisierung und soziale Konflikte gewinnen weltweit an Fahrt (z. B. Birke/Henninger 2012). Seit Beginn der Großen Rezession manifestiert sich insbesondere im Süden Europas Widerstand gegen die Austeritätspolitik der nationalen Regierungen. Doch auch in anderen Teilen Europas haben soziale Unruhen zugenommen, nicht nur im Bereich der Arbeitsbeziehungen. Wie bei allen Protestzyklen wird das Repertoire der Kampfmittel (transnational) durch innovativere Formen von Arbeitskämpfen bereichert, wobei ältere Formen erstmals seit Langem wiederentdeckt werden. Dieser Artikel setzt den Schwerpunkt auf Streiks als eher traditionelles Mittel sozialer Auseinandersetzungen. Der Grund ist, dass die letzte Welle sozialen Protests Ende der 1960er bis Anfang der 1970er Jahre durch einen plötzlichen Anstieg in der Streikaktivität und eine allgemein größere Kampfbereitschaft der Beschäftigten gekennzeichnet war. Dabei wurden in den meisten, wenn auch nicht allen, westeuropäischen Ländern offensive Forderungen und zuweilen auch Kritik an die Gewerkschaftsführungen gerichtet (Crouch/Pizzorno 1978). Es lohnt sich, aus historischer Sicht zu prüfen, ob Streiks heute erneut im Zentrum der sozialen Proteste stehen. Damit wäre auch der Trend des sinkenden Arbeitskampfvolumens der meisten Länder in den letzten beiden Jahrzehnten gebrochen. Selbst wenn ein solcher Bruch trotz des Anstiegs der Arbeitslosigkeit manifest wird, müssen seine Ursachen im Zusammenhang betrachtet werden, da dem Arbeitskampf je nach Zeit und Ort unterschiedliche Bedeutung zukommt.

Im Folgenden werden wir die aktuellsten verfügbaren Streikstatistiken in eine analytische Perspektive der Streikentwicklung in westeuropäischen Ländern seit Krisenbeginn rücken. Der Artikel verfolgt zwei Argumentationsstränge. Zum einen geht es um die Messung von Kampfbereitschaft und kollektivem Widerstand anhand des Streikvolumens. Hier verschlechtert sich insbesondere seit der Wirtschaftskrise die Datenlage. Gleichzeitig wird die Eignung des Streikvolumens als quantitative Annäherung für die vergleichende Analyse in verschiedenen Ländern und Zeitabschnitten fragwürdig (Godard 2011; Gall 2013a). Zum andern erklärt die weite Verbreitung politischer Massenstreiks, ausgelöst durch die neoliberal motivierten Sparmaßnahmen der Regierungen, die relative Stabilität bzw. den Anstieg im Streikaufkommen mehrerer (wenngleich nicht aller) Länder in Westeuropa. Gleichwohl fehlen politische Massenstreiks in traditionell wenig ,streikfreudigen“ Ländern sowie in Nordeuropa (bislang), was jedoch gelegentliche Höchststände in diesen Ländern nicht ausschließt. Allerdings sollten diese Spitzen mit den immer noch üblichen Flächenstreiks vor allem in Verbindung mit Tarifrunden erklärt werden.

Übersetzung aus dem Englischen: Klaus Sticker. 


\section{Sinkende Verfügbarkeit und Zuverlässigkeit von Streikdaten}

Die Erhebung von Streikdaten begann in den meisten westeuropäischen Ländern im letzten Viertel des 19. Jahrhunderts, als die soziale Frage an Bedeutung gewann. In jüngerer Zeit aber zeigen etliche Regierungen und Verwaltungen, insbesondere in den südeuropäischen Krisenländern, kein Interesse mehr an Streikstatistiken. So gibt es in Griechenland etwa seit 1998 ohne Angabe offizieller Gründe keine amtlichen Streikdaten mehr. Das italienische Istituto nazionale di statistica stellte 2009 die Erhebung von Arbeitskampfdaten ein, in Portugal werden amtliche Angaben zu Streikaktivitäten seit 2007 „verschoben“. Ähnlich war 2013 die Erhebung jährlicher Streikdaten in Großbritannien (erneut) bedroht, da das Office for National Statistics erwog, solche Daten wegen Personalkürzungen nicht mehr zu erfassen; zum Zeitpunkt des Entstehens dieses Artikels werden sie nach wie vor erhoben. In Belgien gibt es seit 2002 keine eigene öffentliche Behörde mehr, die Daten zu Streiks erhebt oder ihre Zuverlässigkeit garantiert; als alternative administrative Quelle steht jedoch das Sozialversicherungssystem zur Verfügung. Festzuhalten ist auch, dass bei Eurostat, dem statistischen Amt der Europäischen Union (EU), keine Streikdaten mehr einzusehen sind; stattdessen wird man an LABORSTA weiterverwiesen, die frühere statistische Datenbank der Internationalen Arbeitsorganisation. Zuvor schon wurden die Streikdaten für die meisten EU-Mitgliedstaaten nicht mehr von Eurostat aktualisiert, sodass seit 2008 die nationalen Statistikämter direkt angefragt werden müssen.

Bei der Datenzuverlässigkeit erschweren verschiedene Probleme schlüssige Vergleiche der länderspezifischen Streikentwicklungen. Bedenken hinsichtlich der Verlässlichkeit bestehen aufgrund der je eigenen Methoden der Erfassung von Streikaktivitäten, der (sich ändernden) Kriterien für die Erfassung von Arbeitskämpfen und der fehlenden Unterscheidung zwischen Streiks und Aussperrungen (Lyddon 2007). Ein bemerkenswertes Beispiel hierfür ist Deutschland, wo Streikaktivitäten von der Bundesagentur für Arbeit institutionell untererfasst werden (Dribbusch 2012). Defizite in den Arbeitskampfstatistiken sind ebenso dem bewussten Ausschluss bestimmter Wirtschaftsregionen, Sektoren oder Streiks geschuldet. Insbesondere der Ausschluss von Arbeitskampfmaßnahmen im öffentlichen Sektor und von Generalstreiks beeinträchtigt die Datengenauigkeit zunehmend - eben diese Streiks aber gewinnen in mehreren europäischen Ländern, vor allem seit den 2000er Jahren, an Relevanz (Gall 2013b; Hamann et al. 2013).

Im Folgenden werden Eurostat/LABORSTA-Daten von 1990 bis 2008 mit Arbeitskampfdaten aus nationalen Quellen für die jüngste Zeit (2009 - 2012) und für 19 ausgewählte europäische Länder kombiniert (falls nicht anders angegeben): Belgien (1991-2012), Dänemark, Deutschland ${ }^{2}$, Finnland, Frankreich (1990 - 2011, mit beträchtlichen Da- tenkorrekturen, insbesondere seit 2005), Griechenland (1990 - 98 und unvollständige Daten für 1999 und 2002), Großbritannien, Irland, Italien (1990 - 2009), Luxemburg (1990 - 2008), Malta, Niederlande, Norwegen, Österreich, Portugal (1990-2007), Spanien, Schweden, Schweiz und Zypern.

\section{Keine europaweite Streikwelle seit Krisenbeginn}

Untersuchungen zu den 1990er und frühen 2000er Jahren ergeben ein wiederkehrendes Muster divergierender Streikvolumina in den westeuropäischen Ländern, wobei das Streikaufkommen eher auf niedrigem Niveau stagniert (Scheuer 2006). Dieses Muster unterschiedlicher, länderspezifischer Arbeitskampfvolumina hat sich jüngeren Forschungen zufolge bis heute erhalten. Vergleicht man die 1990er Jahre mit dem Folgejahrzehnt, so bleibt die relative Position der Länder weitgehend konstant (Vandaele 2011). ${ }^{3}$ Gleichwohl ging das Streikaufkommen im ersten Jahrzehnt des neuen Jahrtausends nahezu überall zurück, wobei zunächst deutliche Unstetigkeiten zu verzeichnen waren: Auf Streikspitzen folgten Jahre mit niedrigem Arbeitskampfvolumen. Anders als bei Vandaele 2011 fließen hier in die Betrachtung drei weitere Jahre mit ein - 2010, 2011 und 2012. Vor dem Hintergrund der aktuellen Strukturkrise im Finanzmarkt-Kapitalismus interessiert uns die Frage, ob die Trends im Streikvolumen Bestand haben.

Abbildung 1 zeigt die Entwicklung des einfachen und des gewichteten Mittels des Streikvolumens seit 1990 für bis zu 19 europäische Länder. Ausgehend von den beiden dargestellten Durchschnittswerten bleibt das Streikvolumen Anfang des 21. Jahrhunderts offenbar relativ stabil und geht weder anhaltend noch insgesamt zurück. Erklärungsansätze zu den allgemeinen strukturellen sozioökonomischen und politisch-ideologischen Transformationen hin zu einer neoliberalen Europäisierung können dazu beitragen, die Schwächung der Verhandlungsposition der Beschäftigten und somit der Wirkung von Arbeitskampfmaßnahmen zu verstehen (Piazza 2005; Brandl/Traxler 2010). Da die lokale gewerkschaftliche Organisierung auf Unternehmensebene die Streikwahrscheinlichkeit positiv beeinflusst (Jansen 2014), wären des Weiteren der nahezu allgemein sinkende Organisationsgrad und die erodierende Gewerkschaftsbin-

2 Für den Zeitraum seit 2004 werden die berichtigten Zahlen des Wirtschafts- und Sozialwissenschaftlichen Instituts (WSI) verwendet.

3 Griechenland wurde in diesem Datensatz nicht berücksichtigt, hatte in den 1990er Jahren jedoch das höchste Streikaufkommen der europäischen Länder. 
dung insbesondere in der Fertigung Erklärungen, um das eher niedrige durchschnittliche Streikvolumen vor allem außerhalb Südeuropas zu verstehen.

Betrachtet man allerdings nur das aggregierte Streikvolumen, bleiben sowohl die komplexe, vielschichtige Natur der Streikaktivitäten als auch die sektoralen Unterschiede innerhalb der nationalen Grenzen verdeckt. Die mit der Aggregation verbundene Nivellierung könnte sogar auf eine sektorale Verlagerung der Streikaktivität von der Industrie hin zum Bereich der Dienstleistungen im privaten Sektor und insbesondere im öffentlichen Dienst hindeuten, einhergehend mit einem Wandel in der Logik von Arbeitskampfmaßnahmen (Bordogna/Cella 2002). Aufgrund ihres strategischen Ortes im Produktionssystem verfügen manche Beschäftigte im Dienstleistungssektor - etwa in der Logistik- und Transportbranche - über eine bessere betriebliche Verhandlungsposition. Wenn auch ungleich verteilt, ist diese Verhandlungsstärke mit einem größeren Potenzial gesellschaftlichen Drucks verbunden, sodass sich das Mittel des Arbeitskampfes gezielter einsetzen lässt - mit weniger Streikenden und Streiktagen. Zudem verfügen diese Beschäftigten häufig über Streikerfahrungen aus ihrer früheren Arbeit in der Branche. Angesichts der mangelnden Eignung herkömmlicher Indikatoren für die Untersuchung von Streikaktivitäten im Dienstleistungsbereich wären ein eher qualitativer Ansatz oder andere Interpretationsmodelle geeigneter, wenn es darum geht, die Tertiarisierung von Arbeitskampfmaßnahmen $\mathrm{zu}$ belegen und möglicherweise damit verbundenen Aspekten wie z. B. einer etwaigen Feminisierung nachzugehen.

Betrachtet man den Zeitraum seit Krisenbeginn, dann hat die Ära der Austeritätspolitik zunächst offenbar keinen rasanten Anstieg im Streikvolumen ausgelöst. Dieses Ergebnis sollte nicht sonderlich überraschen, da nicht alle westeuropäischen Länder so stark von Wirtschaftskrise und Austeritätsmantra betroffen sind wie beispielsweise Südeuropa oder Irland. Mindestens ebenso wichtig ist, dass bis 2007 fast alle Länder für die Berechnung des durchschnittlichen Streikvolumens berücksichtigt sind; danach aber sinkt ihre Zahl: entweder, weil einige Länder die Erhebung von Arbeitskampfdaten schlicht eingestellt haben, oder weil aktuelle Streikdaten bei Redaktionsschluss noch nicht verfügbar waren. So gibt es keine aktuellen Daten für Griechenland, Italien und Portugal; im Falle Spaniens wurden die Generalstreiks von 2010 und 2012 in den amtlichen Streikstatistiken bewusst ausgelassen. Auf der Grundlage alternativer Datenquellen, die auch andere Formen von Auseinandersetzungen als ausschließlich Streiks berücksichtigen, kann man Südeuropa zweifellos als das geografische Epizentrum der gegenwärtigen Arbeitskonflikte in Westeuropa bezeichnen (Schmalz/Weinmann 2013, S. 97). Mit anderen Worten: Da (bestimmte) Streikdaten für die genannten Krisenländer nicht vorliegen, lässt sich die relative Stabilität des mittleren Streikvolumens in Westeuropa seit der Wirtschaftskrise mit Fug und Recht anzwei-
ABB.

\section{Durchschnittliches Streikaufkommen in Westeuropa 1990 - 2012}

Angaben in Tagen pro 1000 Beschäftigte

Durchschnitt ohne Griechenland

gewichtetes Mittel

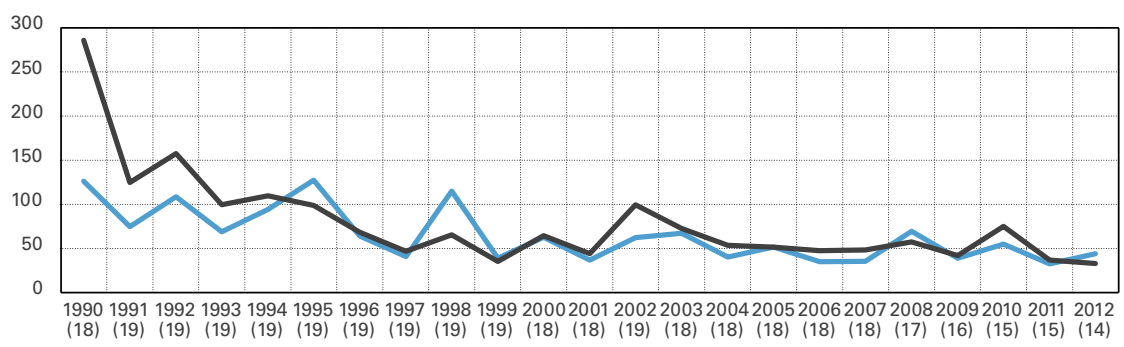

Anmerkung: Die Anzahl der in die Berechnung des Durchschnitts einbezogenen Länder steht in Klammern unter der Jahreszahl.

Quelle: Eurostat und nationale Statistikämter; Darstellung des Autors.

feln. Zudem - und dies zeigt erneut die Unstetigkeit der Streikaktivitäten - weisen die Jahre 2008 und 2010 eine gewisse Zunahme des durchschnittlichen Streikvolumens auf.

Die Unstetigkeit im Streikvolumen verdeutlicht Abbildung 2; dort sind die Länder nach ihrem durchschnittlichen Aufkommen in den 2000er Jahren geordnet. Der erste Balken zeigt die länderübergreifende Veränderung des Aufkommens. Zwei weitere Balken vergleichen das durchschnittliche Volumen im Zeitraum von 2005-2008 sowie in den Jahren seit Verschärfung der Krise (2009-2012). ${ }^{4}$ Ein solcher Abgleich ist für 14 Länder möglich. Die Abbildung zeigt, dass das durchschnittliche Streikvolumen seit der Krise in einigen dieser Länder abgenommen hat. Den stärksten Rückgang verzeichnet Dänemark, wo das Aufkommen 2008 einen Höchstwert hat, bedingt durch eine lang anhaltende Tarifauseinandersetzung im Gesundheitswesen (Jørgenson 2008). Insbesondere der dänische Fall zeigt, wie der gewählte Zeitraum bei einem Länder-Ranking zu Fehlschlüssen führen kann, da die Ergebnisse kurzer Zeiträume stark von außergewöhnlichen Spitzen beeinflusst sind, wenngleich dies für die anderen hier untersuchten Länder weitaus weniger gilt. ${ }^{5}$ Auch in Belgien, Deutschland, Finnland, Großbritannien, Schweden, der Schweiz und scheinbar Spanien ging das Streikvolumen zurück. Würde man jedoch die amtlichen Arbeitskampfdaten durch die Generalstreiks von 2010 und 2012 ergänzen, dann ge-

4 Wir rechnen das Jahr 2008 zur Vorkrisenzeit, da sich die Wirtschaftskrise in den meisten westeuropäischen Ländern erst Ende 2008 manifestierte.

5 Ein Vergleich des Zeitraums 2003-2007 mit 2008-2009 verändert das Gesamtbild im Trend des Streikvolumens außer in Dänemark, Malta und Österreich nicht. 
ABB. 2

Arbeitskampfbedingte Ausfalltage in Westeuropa

Angaben in Tagen pro 1000 Beschäftigte

2001 bis 2010

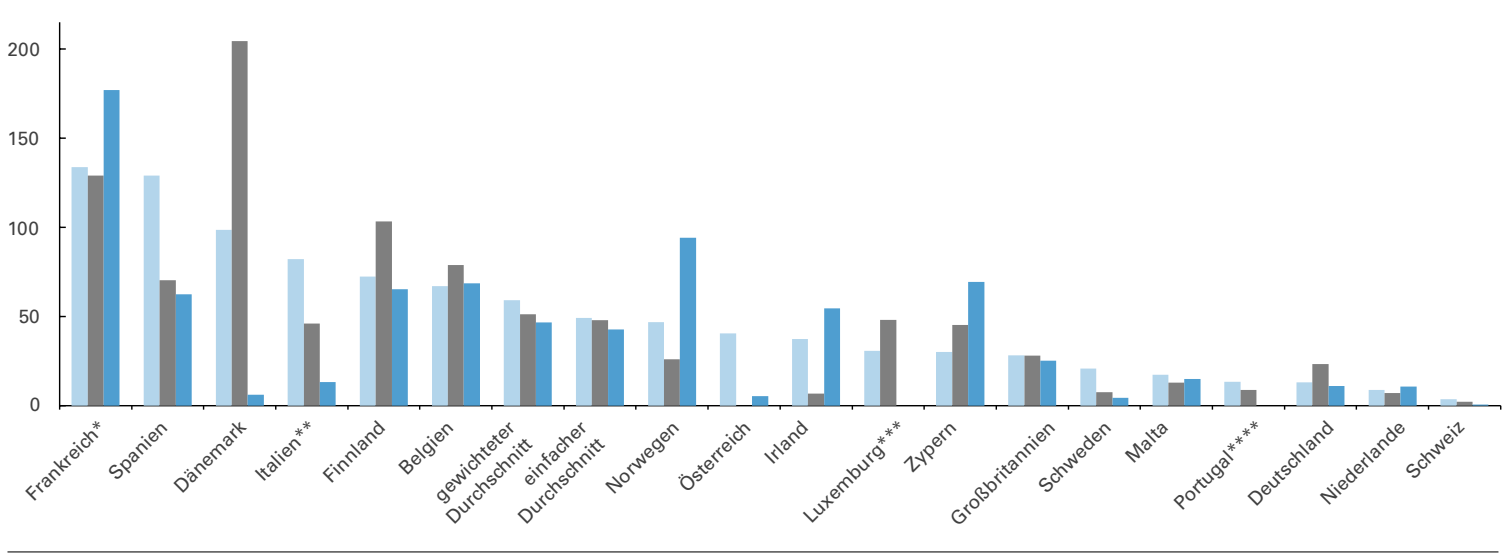

Anmerkung: Geordnet nach den Daten für 2001-2010. Fehlende Jahre: *2012; **2010-2012; ***2009-2012; ****2008-2012

Quelle: Eurostat und nationale Statistikämter; Darstellung des Autors.

hörte Spanien zweifellos zu der Ländergruppe mit einem seit Krisenbeginn gestiegenen Streikvolumen. Mindestens sechs Länder verzeichnen einen Anstieg: Irland, Malta, Niederlande, Norwegen, Österreich und Zypern. Gewiss hinzugefügt werden kann, obwohl für das Jahr 2012 noch keine Streikdaten verfügbar sind, auch Frankreich, geht man vom dortigen Volumenanstieg aus. Kaum Zweifel bestehen auch daran, dass das Streikvolumen in Griechenland, Italien und Portugal insoweit gewachsen ist, dass sie zu dieser Ländergruppe hinzugerechnet werden können. Daher lässt sich resümieren: In elf der hier untersuchten 18 Länder ist das mittlere Streikvolumen seit Krisenausbruch gegenüber dem früheren Vergleichszeitraum gestiegen. ${ }^{6}$ Wenn Streikvolumen und Krise bzw. Austeritätspolitik in Südeuropa offenkundig und direkt miteinander zusammenhängen, so ist dies weniger eindeutig für einige andere Länder. Um dies eingehender zu untersuchen, sollten wir analytisch zwischen verschiedenen Arten von Streiks unterscheiden.

\section{Ermittlung von Spitzen im Streikvolumen}

Insbesondere Massenstreiks beeinflussen das Streikvolumen, entweder infolge eines kurzen Arbeitskampfes mit vielen Streikenden oder eines langen Streiks mit einer relativ kleinen Zahl von Beschäftigten. ${ }^{7}$ Anhand der Arbeitskampfursachen kann man zwischen ökonomischen Flä- chenstreiks und politischen Massenstreiks unterscheiden (Gall 2013b):

- Flächenstreiks sind Arbeitskämpfe innerhalb eines einzelnen Wirtschaftszweigs oder - sektors mit dem vorrangigen Ziel, der Arbeitgeberseite Kosten zu verursachen, um bestimmte Forderungen durchzusetzen.

- Politische Massenstreiks sind entweder allgemeine Arbeitsniederlegungen im öffentlichen Sektor oder die gesamte Wirtschaft bzw. bestimmte Regionen betreffende Ausstände mit dem Ziel, die Regierung zu Zugeständnissen und Kompromissen zu bewegen.

Letztere können heute direkt bzw. mehr oder weniger eng mit der sogenannten europäischen „Staatsschuldenkrise“ und den Protesten der Beschäftigten gegen die Sparpolitik der meisten europäischen Regierungen in Zusammenhang gebracht werden. Diese Verknüpfung mag für Flächenstreiks zwar weniger eindeutig sein, doch kann die Wirtschaftskrise immer noch einen eher indirekten Einfluss haben, da sie der Arbeitgeberseite am Verhandlungstisch eine rigorosere Haltung erlaubt. Außerdem kann die Grenze zwischen Flächen- und politischen Massenstreiks in der Praxis verschwimmen, da sich beide Arten gegenseitig beeinflussen - ein Argument, das bereits Rosa Luxemburg

6 Für die mögliche Entwicklung des Streikaufkommens in Luxemburg seit der Krise lassen sich Aussagen nur schwer treffen.

7 Dies bedeutet nicht, dass kleinere Streiks keine Auswirkungen auf die Arbeitsbeziehungen hätten. 
vorgetragen hat (Wilde 2012, S. 18ff.). Ein Unterschied schließlich betrifft die öffentliche Wahrnehmung von Arbeitskämpfen und Gewerkschaften allgemein sowie die Meinungsbildung dazu; so berichten die Medien über (eintägige) politische Massenstreiks und ihre Nutzung des öffentlichen Raums, beispielsweise durch Straßenproteste und Demonstrationen, eher mehr als über (kleinere) Flächenstreiks (z. B. Martin 2005).

Anders als bei einer Schwerpunktsetzung auf Generalstreiks ermöglicht das Konzept des politischen Massenstreiks ein genaueres Bild auf sektoraler Ebene. Um die Bedeutung von Massenstreiks seit Krisenbeginn einzuschätzen, wird hier das Konzept der Streikwellen verwendet, denn aus historischer Sicht tendieren Streiks stark zu einer zeitlichen und räumlichen Ballung (Kelly 1998). Eine allgemein anerkannte operationale Definition gibt es zwar nicht, doch werden Streikwellen gemeinhin unter Bezug auf die grundlegende Arbeit von Shorter und Tilly (1974; vgl. Biggs 2005, S. 1687) definiert: Danach werden als „Welle“ die Jahre bezeichnet, in denen sowohl die Streikhäufigkeit als auch die Zahl der Streikenden um mindestens $50 \%$ über dem Durchschnitt der vorhergehenden fünf Jahre liegen. Um Streikwellen zu ermitteln - oder Streikspitzen, der wohl angesichts der Entwicklung des mittleren Arbeitskampfvolumens genauere Begriff - wenden wir diese Definition hier auf das Streikaufkommen und die relative Streikbeteiligung an, wobei Letztere eher ein geeignetes Maß für die Erfassung der Streikbereitschaft ist (Friedman 2008, S. 67). Um die ermittelten Spitzen weiter zu interpretieren, analysieren wir die quantitativen Daten eingehender und verwenden ausgewählte Berichte der Europäischen Beobachtungsstelle für Arbeitsbeziehungen (EIRO) der Europäischen Stiftung zur Verbesserung der Lebens- und Arbeitsbedingungen (Eurofound) als Sekundärquelle. ${ }^{8}$ So lassen sich die Höchststände danach kategorisieren, ob sie vorwiegend durch Flächen- oder politische Massenstreiks bedingt sind. Grundsätzlich werden hier nur die Streikspitzen seit Krisenausbruch in einen größeren Zusammenhang gestellt.

Schließlich wären noch zwei Einschränkungen zu machen. Bei der gewählten Methode des gleitenden Durchschnitts könnte die Bedeutung bestimmter Massenstreiks unterschätzt werden, wenn Umfang oder Beteiligung mathematisch nicht groß genug sind, um als Streikspitze zu gelten. Außerdem werden in den amtlichen Arbeitskampfstatistiken bestimmter Länder Massenstreiks systematisch untererfasst, weil Streiks im öffentlichen Sektor oder Generalstreiks ausgeschlossen werden.

\section{Politische Massenstreiks nehmen zu}

Ausgehend von der Bedeutung der Flächenstreiks für die Erklärung von Streikspitzen unterscheiden wir zwischen
TABELLE 1

\section{Streikspitzen in Ländern mit Firmentarifvertragssystemen}

\begin{tabular}{lll}
\hline & 2005-2008 & 2009-2012 \\
\hline Flächenstreiks & 2005: Zypern & 2009: Malta \\
& 2006: Malta*; Zypern & 2012: Zypern** \\
& 2007: Großbritannien* & \\
\hline Politische Massenstreiks & 2006: Großbritannien* & 2009: Irland \\
& & 2011: Großbritannien; Zypern \\
\hline
\end{tabular}

* kein Streikmaximum auf Basis des Streikvolumens.

** keine Daten für Zypern zur Streikbeteiligung 2012 verfügbar.

Quelle: Eurostat und nationale Statistikämter; Berechnungen des Autors.

Ländern mit einem Firmentarifvertragssystem und solchen mit Flächen- bzw. Branchentarifvertragssystemen, weil deren Struktur einen gewissen Einfluss auf das Profil des Streikvolumens hat (Clegg 1976). Auch wenn diese systembedingte Differenzierung nicht der einzige Grund für Unterschiede im Streikvolumen ist, kann die Streikhäufigkeit bei Firmentarifvertragssystemen aufgrund der größeren Anzahl von Verhandlungseinheiten höher sein, wenn auch mit relativ weniger Streikbeteiligten; in Flächentarifvertragssystemen kann die Streikhäufigkeit wiederum niedriger sein, jedoch mit relativ mehr Streikbeteiligten. Die meisten hier untersuchten Länder haben Flächentarifvertragssysteme; für Südeuropa ist unklar, ob die Tarifverhandlungssysteme noch als Systeme gelten können, in denen die Koordinierung der Tarifverhandlungen oberhalb der Unternehmensebene vorherrscht. Solche Zweifel entstehen angesichts der einseitigen, staatlicherseits betriebenen Entkopplung der verschiedenen Tarifverhandlungseinheiten (Molina 2014).

\subsection{Streikspitzen in Firmentarifvertragssystemen}

In Firmentarifvertragssystemen sind branchenweite $\mathrm{Ar}$ beitskämpfe, die zu Spitzen im Streikgeschehen führen, aufgrund der Verhandlungsstrukturen erwartungsgemäß eher selten. Daher lassen sich die Spitzen in Großbritannien und Malta vor der Wirtschaftskrise nur durch den Einfluss der Beteiligung an einigen Flächenstreiks erklären. In Zypern ist, berücksichtigt man sowohl Streikbeteiligung als auch Streikvolumen, nur eine einzige Streikspitze aufgrund branchenweiter Arbeitskämpfe zu verzeichnen. Flächenstreiks erklären auch die Streikspitzen seit Beginn der Krise. Der Höchststand in Malta 2009 kommt durch die zahlreiche Beteiligung an einem Arbeitskampf wegen Personalmangels im Gesundheitswesen und einem wei-

8 Zu den EIROline-Berichten machen wir hier aus Platzgründen keine genauen Quellenangaben; Einzelheiten können beim Verfasser erfragt werden. 
TABELLE 2

\section{Streikspitzen in Ländern mit Flächentarifvertragssystemen}

\begin{tabular}{l|l|l}
\hline & 2005-2008 & 2009-2012 \\
\hline Flächenstreiks & $\begin{array}{l}\text { 2005: Finnland } \\
\text { 2006: Deutschland**; Norwegen } \\
\text { 2008: Dänemark; Deutschland*; } \\
\text { Niederlande }\end{array}$ & $\begin{array}{l}\text { 2010: Finnland; Norwegen } \\
\text { 2011: Niederlande*; } \\
\text { Norwegen; Österreich }\end{array}$ \\
\hline Politische Massenstreiks & 2005: Belgien; Frankreich & $\begin{array}{l}\text { 2010: Frankreich } \\
\text { 2011: Belgien; } \\
\end{array}$ \\
& & 2012: Belgien; Niederlande \\
\hline
\end{tabular}

Anmerkungen: Keine Berechnungen möglich für Griechenland, Luxemburg, Portugal; keine Informationen zur Streikbe teiligung verfügbar für Belgien und Frankreich; einzelne Jahre fehlen für Frankreich (2012) und Italien (2010-12).

* kein Streikmaximum auf Basis des Streikvolumens.

** kein Streikmaximum auf Basis der Streikbeteiligung.

Quelle: Eurostat und nationale Statistikämter, Berechnungen des Autors.

teren wegen der Arbeitsbedingungen für Lehrer zustande. Die Streikspitze 2012 auf Zypern kann den Streiks während der Tarifverhandlungen im Bausektor zugeschrieben werden.

Zwar gab es vor 2009 nur in Großbritannien einen Ausstand, der aufgrund der Streikbeteiligung als politischer Massenstreik gelten kann, doch geht aus Tabelle 1 hervor, dass derartige Arbeitskämpfe für die Erklärung von Streikspitzen seit Krisenbeginn an Bedeutung gewinnen. So ist die Streikspitze in Irland 2009 einem 24-stündigen landesweiten Arbeitskampf im öffentlichen Dienst aus Protest gegen Lohn- und Gehaltskürzungen zuzuschreiben, wodurch auf den öffentlichen Sektor drei Viertel des Streikvolumens entfallen. Außerdem koordinierten die beiden großen Gewerkschaften im öffentlichen Sektor Großbritanniens im Juni und November 2011 Streiks gegen die Rentenreform im öffentlichen Dienst, wobei der November-Streik dem britischen Gewerkschaftsdachverband (TUC) zufolge der größte seit 30 Jahren war. Dennoch hat sich das britische Streikvolumen seit Beginn der Krise im Durchschnitt eher stabilisiert als erhöht. In Zypern schließlich war der Streik der öffentlichen Bediensteten gegen eine zweijährige Nullrunde und die Sparmaßnahmen der erste seit zehn Jahren. Aus all dem folgt: Politische Massenstreiks sind auch in Ländern mit Firmentarifvertragssystemen ein Thema. In allen drei Fällen lässt sich dies mit den einseitig ergriffenen Sparmaßnahmen der Regierungen sowie der Tatsache erklären, dass, anders als im Privatsektor, im öffentlichen Sektor das Flächentarifvertragssystem vorherrscht.

\subsection{Streikspitzen in Flächentarifvertrags- systemen}

In Ländern mit Flächentarifvertragssystemen sind branchenweite Ausstände, die zu Spitzen im Streikgeschehen führen, seit Krisenbeginn nach wie vor von Bedeutung ( $\mathrm{Ta}$ belle 2). Dies gilt insbesondere für die skandinavischen Län- der, was auf die enge Verbindung zwischen Tarifrunden, Friedenspflicht und Streiks hindeutet, wenngleich es erhebliche länderspezifische Unterschiede in der „Toleranzschwelle der Arbeitgeber“ und ihren „rechtlichen Schritten“ gegen wilde Streiks gibt (Stokke/Thörnqvist 2001, S. 256). Hier ist vor allem Norwegen zu nennen, wo Neuverhandlungen über landesweite Tarifverträge im privaten und öffentlichen Sektor häufig zu einem deutlichen Anstieg im Streikvolumen führen, so etwa 2006, 2010 und 2012. Auch in Finnland waren die Tarifverhandlungen von verstärkter Streikaktivität in mehreren Sektoren begleitet, was 2010 zu einer Streikspitze führte.

Dass in jüngerer Zeit keine Massenstreiks zu verzeichnen sind, ist in gewisser Weise Ausdruck der Tatsache, dass Generalstreiks für die Gewerkschaften der skandinavischen Länder kein zentrales Arbeitskampfmittel sind; dennoch gab es in Finnland und Norwegen seit den 1980er Jahren Generalstreiks (Hamann et al. 2013). In dieser Region tragen das Tarifvertragssystem und die hervorgehobene Stellung der Gewerkschaften am Arbeitsmarkt dazu bei, dass Arbeitskampfmittel eher taktisch eingesetzt werden, häufig - bedingt durch die Friedenspflicht - verbunden mit den Tarifrunden. In Südeuropa und Frankreich hingegen sind die Möglichkeiten der Gewerkschaften seit jeher dadurch limitiert, dass die Arbeitgeber sie nicht anerkennen (Hyman/Gumbrell-McCormick 2010, S. 317f.). Daher müssen sich die Gewerkschaften an den Staat wenden, um ihren Forderungen Geltung zu verschaffen, was sich wiederum auf die Wahl der Arbeitskampfmittel auswirkt - vor allem Demonstrations- und politische Massenstreiks. Ob Ausdruck einer eindrucksvollen Geschichte von Mobilisierung und Arbeitskampf oder nicht: Die Vielfalt der Gewerkschaftsideologien und -identitäten erklärt, warum ihre Strategien und Reaktionen auf ähnliche, wenn auch nicht identische Bedingungen kaum monolithisch sind (Martin/Dixon 2010, S. 99ff.).

Nicht nur in Nordeuropa, auch andernorts gab es Streikspitzen durch Flächenstreiks, wie die Zunahmen des Streikaufkommens (2006) und der Streikbeteiligung (2008) in Deutschland zeigen; sie sind weitgehend auf die Warnstreiks in der Metallindustrie zurückzuführen. Es bleibt abzuwarten, ob das deutsche Streikvolumen noch weiter steigen wird, weil die Gewerkschaften ihre Rekrutierungsstrategie in Richtung auf ein „Organisieren am Konflikt" umorientieren (Dribbusch 2011). Auch in der österreichischen Metallindustrie waren die Tarifverhandlungen 2011 von Warnstreiks begleitet. Bei den Streikspitzen in den Niederlanden seit Beginn der Krise ergibt sich kein einheitliches Bild. Der Höchststand 2011 kann angesichts der Arbeitsniederlegungen in den Sozialunternehmen mit einem politischen Massenstreik gegen die spürbaren Sparmaßnahmen der Regierung erklärt werden (CBS 2011). Sparmaßnahmen waren 2012 erneut Ursache für Arbeitskampfmaßnahmen im Bildungswesen, aber mehr als die Hälfte des Streikvolumens war durch Streiks bei Tarifverhandlungen im Reinigungsgewerbe bedingt - das Maximum somit die Folge eines Flächenstreiks (CBS 2012). Ähnlich wie in den skandinavischen 
Ländern sind politische Massenstreiks bei den Gewerkschaften in eher streikarmen Ländern als Arbeitskampfinstrument (derzeit) von sekundärer Bedeutung. Für Frankreich und Belgien sind die Ursachen der Streikspitzen seit Krisenbeginn klarer ersichtlich. In Frankreich gab es 2009 und 2010 General- und Demonstrationsstreiks, die der Forderung nach Maßnahmen der Regierung gegen die Wirtschaftskrise Nachdruck verleihen sollten und gegen die Rentenreformpläne gerichtet waren. Im Nachbarland Belgien waren im darauffolgenden Jahr ein Massenstreik wegen ähnlicher Anlässe und 2012 ein Generalstreik gegen die Sparmaßnahmen der Regierung zu verzeichnen (Gracos 2012). Diese politischen Massenstreiks wirken sich allerdings nur als Stabilisierung des durchschnittlichen Streikvolumens seit Krisenbeginn aus. Auf das übrige Südeuropa schließlich wird hier - Spanien ausgenommen - nicht eingegangen, schlicht, weil keine ausreichenden aktuellen Streikdaten vorliegen. Aber Generalstreiks häufen sich traditionell geografisch im südlichen Teil Europas, wenngleich in Portugal etwas weniger (Hamann et al. 2013). Zweifellos haben politische Massenstreiks, ob Arbeitskämpfe im öffentlichen Sektor oder Generalstreiks, das Streikvolumen in Griechenland, Italien, Portugal und Spanien so beeinflusst, dass Streikspitzen überall dort seit Krisenbeginn auftreten. Dabei wird die Zwangsschlichtung als Mittel der Tarifkonfliktlösung voraussichtlich an Bedeutung gewinnen (Molina 2014).

\section{Fazit}

Vom Mangel an Daten und ihrer Qualität abgesehen ist jegliche Interpretation von Streikdaten eindeutig an das jeweilige Land und den untersuchten Zeitraum gebunden. Des Weiteren ist das aggregierte Streikvolumen als Summe aller Arten von Arbeitskampfmaßnahmen, insbesondere Massenstreiks, ungeeignet, um die unterschiedliche Verwendung des Arbeitskampfmittels Streik durch eine in ihrer strukturellen, organisatorischen und diskursiven Macht heterogene Erwerbsbevölkerung zu erfassen. Unabhängig davon lassen die Arbeitskampfdaten nicht auf einen allgemeinen deutlichen Anstieg des durchschnittlichen Streikvolumens in Westeuropa seit Krisenbeginn schließen. Tatsächlich bilden Flächenstreiks derzeit nicht den Kern des neuen sozialen Protestzyklus; sie sind fester Bestandteil der Kampfmittel von Industriegewerkschaften und traditionell mit den Tarifrunden verbunden - und dies auch weiterhin, das heißt ungeachtet der Wirtschaftskrise, insbesondere in den skandinavischen Ländern. Eine Interpretation der Nivellierung des Streikvolumens wäre daher, dass sie Ausdruck einer Schwächung des Industriegewerkschaftswesens und der damit verbundenen relativ starken Tarifverhandlungsposition (Visser 2012) ist - wenngleich die Streikdrohung nach wie vor Wirkung zeigen mag. Gleichwohl, und dies demonstriert die gewerkschaftliche Mobilisierungsmacht, konzentrieren sich defensive politische Massenstreiks in Südeuropa, insbesondere in Griechenland. Dennoch nehmen diese - gleichermaßen defensiv und meist den öffentlichen Sektor betreffend - auch außerhalb Südeuropas und trotz verschiedener streik- und arbeitsrechtlicher Beschränkungen relativ zu; dies gilt sowohl für Länder mit Firmen- als auch mit Flächentarifvertragssystemen, was das (geringe) Streikvolumen unterstreicht.

Sollten politische Massenstreiks sich weiterhin vorwiegend an die arbeitsrechtlichen Regelungen halten, werden sie wohl weder die anhaltende Ausbreitung neoliberaler Politik bremsen noch zur Neubelebung der Gewerkschaften beitragen. Ihr häufiger und zunehmender Einsatz schließt das
Risiko ein, dass sie ein rein symbolisches Instrument werden (Gall 2013a, S. 20), anstatt die Last der Sparpakete wirksam zu verringern und die politischen Eliten und Autoritäten der postdemokratischen Gesellschaften herauszufordern. Dennoch kann, um mit Rosa Luxemburg zu sprechen, die Beteiligung an Streiks und anderen Arbeitskampfmaßnahmen „das Denken der Menschen über die Wirtschaft, über die Rolle der Regierungen und Märkte und über ihre eigenen Interessen als Beschäftigte, Verbraucher und Bürger“ verändern (Kelly 2011, S. 26). Auch bei allgemein geringer Organisationsdichte (vgl. Sullivan 2010) demonstrieren Gewerkschaften immer noch ihre organisatorische Rolle in der sozialen Mobilisierung, denn sie sind das wichtigste Vehikel für die Planung und Durchführung von Demonstrations- und politischen Massenstreiks. Umgekehrt erwiesen sich andere Formen sozialer Konflikte als kurzlebig, häufig mit nur geringer Beteiligung der Gewerkschaften oder zuweilen auch Ignoranz oder gar Ablehnung seitens derselben (Schmalz/Weinmann 2013, S. 95f.). Gleichwohl und im Großen und Ganzen ist es die Zunahme politischer Massenstreiks, die weitgehend erklärt, weshalb das Streikvolumen seit Krisenbeginn in einer Reihe von Ländern gestiegen oder relativ konstant geblieben ist. Seine weitere Entwicklung und Bedeutung wird zweifellos von den künftigen Strategien von Beschäftigten, Gewerkschaften, Unternehmern und Staat abhängen.

\section{LITERATUR}

Biggs, M. (2005): Strikes as forest fires: Chicago and Paris in the late nineteenth century, in: American Journal of Sociology 110 (6), S. 1684-1714 Birke, P./Henninger, M. (Hrsg.) (2012): Krisen Proteste. Beiträge aus Sozial. Geschichte Online, Berlin

Brandl, B./Traxler, F. (2010): Labour conflicts. A cross-sectional analysis of economic and institutional determinants, 1971-2002, in: European Sociological Review 26 (5), S. 519-540

Bordogna, L./Cella, G. P. (2002): Decline or transformation? Change in industrial conflict and its challenges, in: Transfer 8 (4) S. 585-607

Centraal Bureau voor de Statistiek (CBS) (2011): In 2011 veel stakers bij kortstondige acties, http://www.cbs.nl/nl-NL/menu/themas/arbeid-sociale-zekerheid/ publicaties/artikelen/archief/2012/2012-3615-wm.htm (letzter Zugriff: 06.03.2014)

Centraal Bureau voor de Statistiek (CBS) (2012): Veel stakingsdagen in 2012, http://www.cbs.nl/nl-NL/menu/themas/arbeid-sociale-zekerheid/publicaties/ artikelen/archief/2013/2013-3832-wm.htm (letzter Zugriff: 06.03.2014)

Clegg, H. A. (1976): Trade unionism under collective bargaining. A theory based on comparisons of six countries, Oxford

Crouch, C. /Pizzorno, A. (Hrsg.) (1978): The resurgence of class conflict in Western Europe since 1968, London

Dribbusch, H. (2011): Organisieren am Konflikt: zum Verhältnis von Streik und Mitgliederentwicklung, in: Haipeter, Th./Dörre, K. (Hrsg.): Gewerkschaftliche Modernisierung, Wiesbaden, S. 231-263

Dribbusch, H. (2012): Huge increase in industrial action in 2012, http://www.eurofound.europa.eu/eiro/2013/06/articles/de1306019i.htm (letzter Zugriff: 06.02.2014)

Friedman, G. (2008): Reigniting the labor movement. Restoring means to ends in a democratic labor movement, London

Gall, G. (Hrsg.) (2013a): New forms and expressions of conflict at work, Basingstoke

Gall, G. (2013b): Quiescence continued? Recent strike activity in nine Western European economies, in: Economic and Industrial Democracy 34 (4),

S. 667-691

Godard, J. (2011): What has happened to strikes?, in: British Journal of Industrial Relations 49 (2), S. 282-305

Gracos, I. (2012): Grèves et conflictualité sociale en 2012, Brüssel

Hamann, K./Johnston, A./Kelly, J. (2013): Striking concessions from governments. The success of general strikes in Western Europe, 1980-2009, in: Comparative Political Studies 46 (1), S. 23-41 
Hyman, R./Gumbrell-McCormick, R. (2010): Trade unions, politics and parties: Is a new configuration possible?, in: Transfer 16 (3), S. 315-331

Jansen, G. (2014): Effects of union organization on strike incidence in EU companies, in: Industrial and Labor Relations Review 67 (1), S. 60-85

Jørgensen, C. (2008): Longest strike in public sector ends with pay settlement, http://www.eurofound.europa.eu/eiro/2008/04/articles/dk0804029i.htm (letzter Zugriff: 06.03.2014)

Kelly, J. (1998): Rethinking industrial relations. Mobilisation, collectivism and long waves, London

Kelly, J. (2011): Theories of collective action and union power, in: Gall, G./ Wilkinson, A./Hurd, R. W. (Hrsg.): The international handbook of labour unions. Responses to neo-liberalism, Cheltenham, S. 13-28

Lyddon, D. (2007): Strike statistics and the problems of international comparison, in: van der Velden, S./Dribbusch, H./Lyddon, D./Vandaele, K. (Hrsg.): Strikes around the world, 1968-2005. Case-studies of 15 countries, Amsterdam, S. 24-39

Martin, A. W. (2005): Addressing the selection bias in media coverage of strikes. A comparison of mainstream and specialty print media, in: Research in Social Movements, Conflicts, and Change 26, S. 143-178

Martin, A. W./Dixon, M. (2010): Changing to win? Threat, resistance, and the role of unions in strikes, 1984-2002, in: American Journal of Sociology 116 (1), S. $93-129$

Molina, O. (2014): Self-regulation and the state in industrial relations in Southern Europe: back to the future, in: European Journal of Industrial Relations 20 (1), S. $21-36$

Piazza, J. A. (2005): Globalizing quiescence. Globalisation, union density and strikes in 15 industrialized countries, in: Economic and Industrial Democracy 26 (2), S. $289-314$

Scheuer, S. (2006): A novel calculus? Institutional change, globalization and industrial conflict in Europe, in: European Journal of Industrial Relations 12 (2), S. $143-164$
Schmalz, S./Weinmann, N. (2013): Zwei Krisen, zwei Kampfzyklen: Gewerkschaftsproteste in Westeuropa im Vergleich, in: Schmalz, S./Dörre, K. (Hrsg.): Comeback der Gewerkschaften? Machtressourcen, innovative Praktiken, internationale Perspektiven, Frankfurt a. M., S. 76-98

Shorter, E./Tilly, C. (1974): Strikes in France, New York

Stokke, T. A./Thornqvist, C. (2001): Strikes and collective bargaining in the Nordic countries, in: European Journal of Industrial Relations 7 (3), S. 245-267 Sullivan, R. (2010): Labour market or labour movement? The union density bias as barrier to labour renewal, in: Work, Employment \& Society 24 (19), S. $145-156$

Vandaele, K. (2011): Sustaining or abandoning "social peace"? Strike development and trends in Europe since the 1990s, Working Paper 2011.05, Brüssel Visser, J. (2012): The rise and fall of industrial unionism, in: Transfer 18 (2), S. $129-141$

Wilde, F. (2012): Einleitung: Die Rückkehr des politischen Streiks, in: Gallas, A./Nowak, J./Wilde, F. (Hrsg.): Politische Streiks im Europa der Krise, Hamburg, S. 7-21

\section{AUTOR}

KURT VANDAELE, Dr., ist Senior Researcher am Europäischen Gewerkschaftsinstitut (EGI) in Brüssel. Arbeitsschwerpunkte: Erneuerung der Gewerkschaften, Arbeitskämpfe.

kvandaele@etui.org 